\title{
A bone remodelling model based on generalised thermodynamic potentials and optimisation applied to a trabecula with cyclic loading
}

\author{
F.V. Tormena, E.G.F. Mercuri* and M.B. Hecke \\ Bioengineering Group, Federal University of Paraná, Curitiba, Brazil
}

\begin{abstract}
.
BACKGROUND: Bone diseases caused by an imbalance of bone turnover represent a major public health concern worldwide. Studies involving bone remodelling mechanisms can assist in the treatment of osteoporosis, osteopenia and in cases of fractures. In recent decades several authors have developed bone remodelling models.

AIM: The aim of this study is to propose a model based on the thermodynamic framework to describe the process of bone remodelling. A secondary aim is to model a trabecula subjected to cyclic loading and calibrate the model with experimental data.

METHODS: Thermodynamic potentials are used to generate the functions of state based on internal scalar variables. The evolution of the variables in time is determined by dissipation potentials, which are created through the use of convex analysis. Constitutive equations are solved with mathematical programming algorithms and the numerical implementation of this theory uses the Finite Elements Method for spatial discretization.

RESULTS: The proposed theory was applied to a one-dimensional example, and two situations (an undamaged material and an initially damaged material) were simulated. The one-dimensional example shows a microscopic view of a trabecula under the influence of a growing load cycle throughout 1200 days. This dynamic process may represent the rehabilitation of an athlete, starting with light exercises up to a very heavy physical activity.

CONCLUSIONS: The model was able to represent one bone remodelling cycle in the trabecula. Although it is not yet possible to obtain an experimental curve of a traction test in vivo, the in silico model showed a process of damage that is similar to the static test of the literature. The results also suggest a modification in the equation adopted for the Helmholtz potential shown here. This study presents a consistent thermodynamic formalism for bone remodelling, which may allow further contributions as the incorporation of chemical reactions, mass transference and anisotropic damage.
\end{abstract}

Keywords: Bone remodelling, thermodynamics, internal scalar variables, convex analysis, mathematical programming

${ }^{*}$ Corresponding author: E.G.F. Mercuri, Universidade Federal do Paraná (UFPR), Setor de Tecnologia, Centro Politécnico - Bloco Lame/Cesec - Jd Américas, CEP 81531-990, Curitiba, Paraná, Brasil. E-mail: emiliomercuri@gmail.com.

\section{Introduction}

Bone diseases caused by an imbalance of bone turnover represent a major public health concern worldwide. Studies involving bone remodelling mechanisms can assist in the treatment of osteoporosis, osteopenia and in cases of fractures. Changes in the 
homeostasis of the dynamic system of the bone are affected by mechanical stimuli [57, 58]. Groups of specialized cells, such as osteoblasts and osteoclasts, are responsible for, respectively, forming and resorbing bone matrix [2].

Several authors have developed bone remodelling models in recent decades. Cowin and Hegedus have used the strain tensor as the mechanical signal that is the driving factor of bone adaptation, and the fabric tensor was introduced for describing the microstructure of cancellous bone remodelling [30, 57]. Some authors have used strain energy density as the stimuli for bone remodelling [44]. Frost described the difference between modelling and remodelling [19-21] and $[17,56]$ related to Young's modulus with the apparent density. Prendergast and Taylor [41] suggested that the bone adapts itself to attain an optimal strength by regulating the damage generated in its microstructural elements.

In the study by Jacobs et al. [6], an anisotropic evolution of the elasticity tensor was presented, and bone resorption was assumed to be a cumulative damage process. Cowin and Hegedus [56] proposed an adaptive elasticity theory, while Ramtani and Zidi [54] included the contribution of bone microdamage as a stimulus. Prendergast and Taylor [41] suggested that microcracks in the bone could be correlated with cell activation. García-Aznar et al. [23] considered a bone remodelling model that could simulate microdamage growth and repair in a coupled way. According to McNamara and Prendergast [33], there is a consensus among researchers that microdamage is an important stimulus for bone remodelling.

In this study, the thermodynamic state variables are two internal variables that represent the loss of stiffness (damage) and the gain of stiffness in bone mechanics. The evolution of those variables follows an associated potential that is related to the choice of the Helmholtz free energy, according to $[3,30,31]$. The gradient of the conjugated Helmholtz potential provides the direction for the evolution of the internal variables. This associated contribution is important for the development of a consistent mathematical model for the remodelling process.

The numerical implementation through optimisation follows the principles of continuum damage mechanics and the plasticity theory $[1,25,53]$. Convex analysis techniques were used in the development of the mathematical formulation based on other studies $[9,12,16,36,38,46,48,62]$.
The aim of this study is to propose a mathematical formulation based on thermodynamics to describe the process of bone remodelling. The aim of the numerical simulation is to model a simple trabecula geometry subjected to cyclic loading. The experimental curve of a traction test [8] is used to calibrate the model in the damage phase. One fundamental purpose of the model is to create a posterior part of the stress-strain diagram, which can restore part of the original stiffness while simulating a hypothetical remodelling process.

Following the hypothesis of several authors [23, $33,35,41,54]$ the damage level was chosen to be the mechanical stimulus for remodelling. Once one material point reaches a critical damage, remodelling begins, i.e., an optimisation starts the restoring process of the original stiffness.

The dissipation potential describes how variables of state evolve over time. As an analogy of the classical plasticity theory, which defines a yielding function as a restriction for elastic stress, in this study the remodelling functions related to bone formation and degradation define a region of admissible generalised thermodynamic forces. Such formalism generates a nonlinear programming problem that is used to define bone loss or bone formation at each point of a finite element model. Hence, the material properties are updated, characterizing an increase or decrease in bone density.

\subsection{Bone remodelling}

Bones are responsible for the global structural stiffness and strength of the body. They can grow, modify and self-renew over the course of life through a process called bone remodelling. One of the most important characteristics of the bone is its self-adaptation capability, which consists in the change in microstructure and material properties according to the mechanical environment. Remodelling occurs throughout life and it plays a fundamental role in the evolution of the bone microstructure, and consequently, in the adaptation of the structural properties and the repair of microdamage [35].

According to Turner [5], there are three rules for bone adaptation to mechanical stimuli. First, bone adaptation is driven by dynamic - rather than static - loading. Second, only a short duration of mechanical loading is necessary to initiate an adaptive response. Extending the duration of the loading has a diminishing effect on further bone adaptation. The last rule is 
that bone cells accommodate to a customary mechanical loading environment, making them less responsive to routine loading signals.

Four distinct cells perform the local formation, resorption and maintenance of the bone: osteoblasts, osteoclasts, osteocytes, and bone lining cells [27]. The coupling process is based on the separate actions of bone-degrading cells (osteoclasts) and bone-forming cells (osteoblasts) [29], and it begins on the surface of the bone with osteoclasts isolating the area, while adhering to the bone matrix and creating an irregular board in the bone/osteoclast interface. After that, the osteoclasts secrete an acid into the micro-environment and dissolve the organic and inorganic bone matrix [45].

After the degradation process, some osteoblasts move to the same location and begin to form a new tissue. During the formation stage some osteoblasts remain "buried" in the bone matrix and turn into osteocytes. When osteoblasts stop synthesizing bone, they can either die through the apoptosis process or be transformed into cells that cover the new bone surface (lining cells). Those surface cells become highly attached to the bone matrix through the network of canaliculi [45]. This whole process is known as the basic metabolic unit (BMU) [51].

In recent studies, it is considered that bone remodelling is not only a process of adaptation to external loading, but also a consequence of microdamage removal $[4,7,41,49,50,59]$.

\section{Proposal of a constitutive model for bone remodelling using internal scalar variables}

A bone remodelling model should be capable of describing the bone degradation and bone formation processes in the presence of stimuli.

Some studies [6, 11, 15, 22, 55] present a mechanical approach for bone remodelling using damage, plastic deformation and bone repair as variables, but none of them has considered bone degradation and bone formation in the same model.

In this study, the elasto-plasticity theory presented by $[38,39,46,47,62]$, which uses the convex analysis concepts of [26, 52] and of thermodynamics [24, 43], is adapted to describe the law of bone remodelling, which is characterised by the action of a dissipative process (bone degradation) and of an energy gain (bone formation).
The behaviour of the material is described by the definition of two thermodynamic potentials, an energy function (potential for forces) and a dissipation function (potential for the irreversible phenomena). The constitutive relations are directly derived from the free energy (Helmholtz potential), and this free energy function is obtained from the Clausius-Duhem inequality.

The formulation used is based on generalised thermodynamics, in which internal scalar variables are introduced on the basis of constitutive relations to describe an inelastic process that occurs on materials $[10,37]$. The construction of the constitutive equation was performed under the consideration that equations can be solved by using some mathematical programming algorithms [14]. The spatial discretization is made by the Finite Element Method.

Parameters and curves of experimental tests from the literature were used to calibrate the model; some of the relevant studies were done by $[8,18,32,35$, $40,42]$.

\subsection{State variables}

The process of bone loss and repair, which is an internal process that occurs in the bone structure, will be completely defined for the isothermal case if the deformation tensor $\boldsymbol{\varepsilon}$ (observable variable) and a set of $N$ internal scalar variables $\left\{\alpha_{1}, \alpha_{2}, \alpha_{3}, \ldots, \alpha_{N}\right\}$ are known. Some of those variables, which are called $\left\{\alpha^{d}\right\}_{i}$, will be used to describe the stiffness degradation process (microcracking, bone loss), while the other variables, called $\left\{\alpha^{h}\right\}_{j}$, will be used to describe the stiffness gain process (formation, bone gain).

The bulk density $\rho$ is affected by the same mechanisms of loss and repair, and is defined by:

$$
\rho=\rho\left(\left\{\alpha^{d}\right\}_{i},\left\{\alpha^{h}\right\}_{j}\right)
$$

The current thermodynamic state can be described by the Helmholtz free energy density $\psi$, which is a proper, strictly convex function of both observable and internal variables.

$$
\psi=\psi\left(\varepsilon,\left\{\alpha^{d}\right\}_{i},\left\{\alpha^{h}\right\}_{j}\right)
$$

where $\boldsymbol{\varepsilon}$ is the admissible strain tensor and $\left\{\alpha^{d}\right\}_{i},\left\{\alpha^{h}\right\}_{j}$ are the internal variables used to describe the loss and gain of stiffness, respectively. 
For the linear elastic material, the Helmholtz free energy density $\psi$ is defined by the following:

$$
\psi\left(\boldsymbol{\varepsilon},\left\{\alpha^{d}\right\}_{i},\left\{\alpha^{h}\right\}_{j}\right)=\frac{1}{2 \rho} \boldsymbol{D}\left(\left\{\alpha^{d}\right\}_{i},\left\{\alpha^{h}\right\}_{j}\right) \boldsymbol{\varepsilon} \cdot \boldsymbol{\varepsilon}
$$

where $\boldsymbol{D}\left(\left\{\alpha^{d}\right\}_{i},\left\{\alpha^{h}\right\}_{j}\right)$ is a fourth order tensor called elastic tensor, which incorporates the effect of the loss and gain of stiffness, and the symbol (.) represents the internal product.

The dual variables, stress $\sigma$ and the set of thermodynamic forces $\left\{A^{d}\right\}_{i},\left\{A^{h}\right\}_{j}$ associated with the sets of internal variables $\left\{\alpha^{d}\right\}_{i},\left\{\alpha^{h}\right\}_{j}$ are derived from the density free energy $\psi$ according to the state laws as follows:

$$
\begin{gathered}
\boldsymbol{\sigma}\left(\boldsymbol{\varepsilon}, \alpha^{d}, \alpha^{h}\right)=\rho\left(\alpha^{d}, \alpha^{h}\right) \frac{\partial \psi\left(\boldsymbol{\varepsilon}, \alpha^{d}, \alpha^{h}\right)}{\partial \boldsymbol{\varepsilon}} \\
=\boldsymbol{D}\left(\alpha^{d}, \alpha^{h}\right) \boldsymbol{\varepsilon} \\
A^{d}\left(\boldsymbol{\varepsilon}, \alpha^{d}, \alpha^{h}\right)=-\rho\left(\alpha^{d}, \alpha^{h}\right) \frac{\partial \psi\left(\boldsymbol{\varepsilon}, \alpha^{d}, \alpha^{h}\right)}{\partial \alpha^{d}} \\
=-\frac{1}{2} \frac{\partial \boldsymbol{D}\left(\alpha^{d}, \alpha^{h}\right)}{\partial \alpha^{d}} \boldsymbol{\varepsilon} \cdot \boldsymbol{\varepsilon} \\
-\frac{1}{2} \frac{1}{\rho\left(\alpha^{d}, \alpha^{h}\right)} \frac{\partial \rho\left(\alpha^{d}, \alpha^{h}\right)}{\partial \alpha^{d}} \boldsymbol{D}\left(\alpha^{d}, \alpha^{h}\right) \boldsymbol{\varepsilon} \cdot \boldsymbol{\varepsilon} \\
A^{h}\left(\boldsymbol{\varepsilon}, \alpha^{d}, \alpha^{h}\right)=\rho\left(\alpha^{d}, \alpha^{h}\right) \frac{\partial \psi\left(\boldsymbol{\varepsilon}, \alpha^{d}, a^{h}\right)}{\partial \alpha^{h}} \\
=\frac{1}{2} \frac{\partial \boldsymbol{D}\left(\alpha^{d}, \alpha^{h}\right)}{\partial \alpha^{h}} \boldsymbol{\varepsilon} \cdot \boldsymbol{\varepsilon} \\
+\frac{1}{2} \frac{1}{\rho\left(\alpha^{d}, \alpha^{h}\right)} \frac{\partial \rho\left(\alpha^{d}, \alpha^{h}\right)}{\partial \alpha^{h}} \boldsymbol{D}\left(\alpha^{d}, \alpha^{h}\right) \boldsymbol{\varepsilon} \cdot \boldsymbol{\varepsilon}
\end{gathered}
$$

To simplify, let $i=1$, therefore $\alpha^{d}, \alpha^{h}$ will represent $\left\{\alpha^{d}\right\}_{i},\left\{\alpha^{h}\right\}_{j}$ and also $A^{d}, A^{h}$ to represent the sets $\left\{A^{d}\right\}_{i},\left\{A^{h}\right\}_{j}$.

The dual convex function or the conjugated function $\psi_{C}$ of $\psi$ is obtained by the Legendre-Fenchel transform as follows:

$$
\begin{aligned}
& \frac{1}{\rho\left(\alpha^{d}, \alpha^{h}\right)} \psi_{C}\left(\boldsymbol{\sigma}, A^{d}, A^{h}\right) \\
& =\sup _{\boldsymbol{\varepsilon}, \alpha^{d}, \alpha^{h}}\left[\boldsymbol{\sigma} \cdot \boldsymbol{\varepsilon}-A^{d} \alpha^{d}+A^{h} \alpha^{h}\right. \\
& \left.\quad-\rho\left(\alpha^{d}, \alpha^{h}\right) \psi\left(\varepsilon, \alpha^{d}, \alpha^{h}\right)\right]
\end{aligned}
$$

and may be used to define the state laws in an equivalent way as follows:

$$
\begin{aligned}
\boldsymbol{\varepsilon} & =\frac{1}{\rho\left(\alpha^{d}, \alpha^{h}\right)} \frac{\partial \psi_{C}\left(\boldsymbol{\sigma}, A^{d}, A^{h}\right)}{\partial \boldsymbol{\sigma}} \\
\alpha^{d} & =-\frac{1}{\rho\left(\alpha^{d}, \alpha^{h}\right)} \frac{\partial \psi_{C}\left(\boldsymbol{\sigma}, A^{d}, A^{h}\right)}{\partial A^{d}} \\
\alpha^{h} & =\frac{1}{\rho\left(\alpha^{d}, \alpha^{h}\right)} \frac{\partial \psi_{C}\left(\sigma, A^{d}, A^{h}\right)}{\partial A^{h}}
\end{aligned}
$$

For the purpose of obtaining the strains $\boldsymbol{\varepsilon}, \alpha^{d}$ and $\alpha^{h}$, the sets of internal variables are used to describe the loss and gain of stiffness.

\subsection{Evolution laws}

In order to complete the constitutive equations, the evolution laws of the internal variables (loss and gain of stiffness) must be added to the state laws. The thermodynamics theory for irreversible processes provides a guideline and some restrictions to obtain the evolution laws, so that the second principle of thermodynamics must be satisfied for any evolution of the material, in other words, so that the dissipation is non-negative [28].

Similarly to the plasticity theory, two models can be constructed. The first model, on which this paper is based, addresses an "associated" law for which the normality rule is valid. The second model, with a "non-associated" law, involves the construction of a double potential function of two variables. The result is biconvex and satisfies the generalised inequality from Fenchel, whose proposal is found in the studies of $[13,34]$.

In this study, the evolution laws of the internal variables are based on the framework of convex analysis and more specifically on Theorems and Lemmas stated by [62].

Let us consider the region of admissible thermodynamic forces $P$, which is defined as follows:

$$
P=\left\{\left(\sigma, A^{d}, A^{h}\right) / f\left(\sigma, A^{d}, A^{h}\right) \leq 0\right\}
$$

However, the bone remodelling model proposed in this study does not induce dissipation on the tension field, since the bone has elastic behaviour. Thus, it is considered that the region of admissible thermodynamic forces $P_{A}$ defined in Equation 12 is the 
projection of region $P$ in the area of thermodynamics forces $\boldsymbol{A}=\left(A^{d}, A^{h}\right)$.

This way, $P_{A}$ is defined by the following:

$$
P_{A}=\left\{\left(A^{d}, A^{h}\right) / f\left(\sigma, A^{d}, A^{h}\right) \leq 0\right\}
$$

which is assumed to be a closed, convex and nonempty set.

Function $f(\boldsymbol{\sigma}, \boldsymbol{A})$ is called remodelling function, and it is equivalent to the yield function that is used in the plasticity theory.

There is a "dead zone" where even the degradation or gain of elastic stiffness does not occur, and this zone appears when $f(\boldsymbol{\sigma}, \boldsymbol{A})<0$; whereas the behaviour of a loss and gain of stiffness appears when $f(\boldsymbol{\sigma}, \boldsymbol{A})=0$. The situation of $f(\boldsymbol{\sigma}, \boldsymbol{A})>0$ is unacceptable.

Let $\chi$ be the support function of $P_{A}$ :

$$
\chi(\dot{\boldsymbol{\alpha}})=\sup _{A^{*} \in P_{A}}\left(A^{*} \cdot \dot{\boldsymbol{\alpha}}\right)
$$

where $\dot{\alpha}$ is the rate for internal variables and, in the context of elasto-plasticity, $\chi$ is the dissipation function, and in this study it represents the energy dissipated or introduced in the system. This function is conjugated to the indicator function of $P_{A}, \operatorname{Ind}_{P_{A}}$, as defined by the following:

$$
\operatorname{Ind}_{P_{A}}(\boldsymbol{A})= \begin{cases}0, & \text { if } \boldsymbol{A} \in P_{A} \\ \infty, & \text { if } \boldsymbol{A} \notin P_{A},\end{cases}
$$

and it is: convex, positively homogeneous, lower semicontinuous. Function $\chi$ must satisfy the inequality $(\chi(\dot{\alpha}) \leq 0)$ and must contain the origin, being equal to zero in it, $\chi(0)=0$. Therefore, it follows that:

$$
\dot{\boldsymbol{\alpha}} \in \partial \operatorname{Ind}_{P_{A}} \leftrightarrow \boldsymbol{A} \in \partial \chi(\dot{\boldsymbol{\alpha}})
$$

Equation 15 allows the establishment of two equivalent forms of the evolution law. It is necessary to complete the theory and to write the evolution law using a Lagrange multiplier $\lambda$; thus, it follows that:

$$
\dot{\alpha} \in \partial \operatorname{Ind}_{P_{A}} \leftrightarrow \exists \lambda \geq 0 \forall \dot{\alpha} \in \partial f(\boldsymbol{\sigma}, \boldsymbol{A})
$$

Equation 16 represents a generalisation of the normality law.

The definition of the convex region of admissible thermodynamic forces must be made based on some experimental parameters; therefore, the boundary of the region that limits the thermodynamic forces values (or a composition of them) determines the strength change (loss or gain of strength).
For optimum solution of the nonlinear programming problem the Karush-Kuhn-Tucker conditions must be satisfied. They can be written as follows:

$$
\lambda \geq 0, f \leq 0, \dot{\lambda} f=0
$$

\subsection{Incremental formulation for damage-remodelling constitutive equations}

For the implementation of the proposed formulation, it is necessary to write the continuous equations in a discrete form.

Consider the time interval $[0, T]$, which defines a damage-repair process. This time interval can be divided into $N$ sub-intervals, with size $\Delta T$. The size of the time sub-intervals doesn't need to be the same.

$$
\begin{gathered}
0=t_{0}<t_{1}<t_{2}<\cdots<t_{n-1}<t_{n}<t_{n+1}< \\
\ldots<t_{N-2}<t_{N-1}<t_{N}=T
\end{gathered}
$$

Let us assume that the value of any variable $\beta$ in time $t_{n}$ is $\beta\left(t_{n}\right)=\beta_{n}$ and that $\Delta \beta=\beta_{n}-\beta_{n-1}$. The solution of the problem on interval $[0, T]$ is given by solving $N$ additional problems in finite iterative time steps.

This approach is considered to be an implicit solution method that guarantees the convergence and stability of the solution. The following expressions were written after applying Euler's regressive approximations:

$$
\begin{gathered}
\boldsymbol{\varepsilon}_{t+\Delta t}=\frac{1}{\rho\left(\alpha_{t}^{d}, \alpha_{t}^{h}\right)} \frac{\partial \psi_{C}\left(\sigma_{t+\Delta t}, A_{t+\Delta t}^{d}, A_{t+\Delta t}^{h}\right)}{\partial \boldsymbol{\sigma}} \\
\alpha_{t+\Delta t}^{d}=\alpha_{t}^{d}+\Delta \alpha^{d} \\
\alpha_{t+\Delta t}^{h}=\alpha_{t}^{h}+\Delta \alpha^{h} \\
\sigma_{t+\Delta t}=\sigma_{t}+\Delta \sigma \\
A_{t+\Delta t}^{d}=A_{t}^{d}+\Delta A^{d} \\
A_{t+\Delta t}^{h}=A_{t}^{h}+\Delta A^{h}
\end{gathered}
$$

In this study, the formulation proposed by [38] for the elasto-plastic model is adapted to a constitutive damage-remodelling model. Potential $j_{C}$, in incremen- 
tal form is defined by the following:

$$
\begin{gathered}
j_{C}\left(\Delta \boldsymbol{\sigma}, \Delta A^{d}, \Delta A^{h}\right) \\
=\frac{\psi_{C}\left(\sigma+\Delta \sigma, A^{d}+\Delta A^{d}, A^{h}+\Delta A^{h}\right)}{\rho\left(\alpha^{d}+\Delta \alpha^{d}, \alpha^{h}+\Delta \alpha^{h}\right)} \\
-\Delta \sigma \cdot \nabla_{\sigma} \frac{\psi_{C}\left(\sigma, A^{d}, A^{h}\right)}{\rho\left(\alpha^{d}, \alpha^{h}\right)} \\
-\Delta A^{d} \cdot \nabla_{A^{d}} \frac{\psi_{C}\left(\sigma, A^{d}, A^{h}\right)}{\rho\left(\alpha^{d}, \alpha^{h}\right)} \\
-\Delta A^{h} \cdot \nabla_{A^{h}} \frac{\psi_{C}\left(\sigma, A^{d}, A^{h}\right)}{\rho\left(\alpha^{d}, \alpha^{h}\right)} \\
-\frac{\psi_{C}\left(\sigma, A^{d}, A^{h}\right)}{\rho\left(\alpha^{d}, \alpha^{h}\right)}
\end{gathered}
$$

for which $\Delta \sigma, \Delta A^{d}, \Delta A^{h}$ are, respectively, the increments of stress, thermodynamic force of loss of stiffness and thermodynamic force of gain of stiffness. The following is the conjugate:

$$
\begin{aligned}
& j\left(\Delta \boldsymbol{\varepsilon}, \Delta \alpha^{d}, \Delta \alpha^{h}\right) \\
& =\sup _{\Delta \boldsymbol{\sigma}, \Delta A^{d}, \Delta A^{h}}\left\{\Delta \boldsymbol{\sigma} \cdot \Delta \boldsymbol{\varepsilon}-\Delta A^{d} \Delta \alpha^{d}\right. \\
& \left.\quad+\Delta A^{h} \Delta \alpha^{h}-j_{C}\left(\Delta \boldsymbol{\sigma}, \Delta A^{d}, \Delta A^{h}\right)\right\}
\end{aligned}
$$

which can be condensed as follows:

$$
\begin{aligned}
\left(\Delta \boldsymbol{\sigma}, \Delta A^{d}, \Delta A^{h}\right) & =j\left(\Delta \boldsymbol{\varepsilon}, \Delta \alpha^{d}, \Delta \alpha^{h}\right) \Leftrightarrow \\
\left(\Delta \boldsymbol{\varepsilon}, \Delta \alpha^{d}, \Delta \alpha^{h}\right) & =j_{C}\left(\Delta \boldsymbol{\sigma}, \Delta A^{d}, \Delta A^{h}\right)
\end{aligned}
$$

The Equation defined on 26 is satisfied at the end of each time step. The peculiarity of this formulation is that the solution takes the form of a minimisation process of a convex function in a convex set; therefore, it is a convex optimisation problem.

The increments of tension and thermodynamic forces $\Delta \sigma, \Delta A^{d}, \Delta A^{h}$ could be determined from increments of the deformation and internal variables $\Delta \boldsymbol{\varepsilon}, \Delta \alpha^{d}, \Delta \alpha^{h}$ by constitutive relation 26 .

According to [52], Equation 28 expresses the necessary and sufficient condition that the generalised stress ( $\Delta \sigma, \Delta A^{d}, \Delta A^{h}$ ) is found to be the solution of the problems.

$$
\left(\Delta \alpha^{d}, \Delta \alpha^{h}\right) \in \partial \operatorname{Ind}_{P_{A}}\left(A_{t+\Delta t}^{d}, A_{t+\Delta t}^{h}\right)
$$

\section{Proposed formulation for a one-dimensional case}

The proposed formulation for the one-dimensional case and the construction of the constitutive equations are based on the choice of internal variables and thermodynamic potentials. One of the most important characteristics of the presented potentials is the convexity, but according to [31], in some cases convexity is not implicated in the whole domain, which can be solved by imposing admissible domain restrictions on the formulation.

\subsection{One-dimensional formulation with internal scalar variables of the gain and loss of stiffness}

The remodelling function for the one-dimensional case is defined by a function of dual variables. Therefore, the remodelling function considers the stresses $\sigma$ and the thermodynamic forces $\left(A^{d}, A^{h}\right)$ and may be written as $f=f\left(\sigma, A^{d}, A^{h}\right)$.

According to the remodelling function, the admissible region is defined by the following:

$$
P=\left\{\left(\sigma, A^{d}, A^{h}\right) / f\left(\sigma, A^{d}, A^{h}\right) \leq 0\right\}
$$

One of the assumptions of the proposed model is that there is no dissipation in the tension field. This way, the admissible region $P_{A}$ becomes a projection of the $P$ region in the thermodynamic force domain and is defined as follows:

$$
P_{A}=\left\{\left(A^{d}, A^{h}\right) / f\left(\sigma, A^{d}, A^{h}\right) \leq 0\right\}
$$

\subsubsection{Stage of material loss of stiffness}

In the stage of loss of stiffness, damage effects appear in the material, therefore, $\alpha^{d} \neq 0$ and $\alpha^{h}=0$. The diagram in Fig. 1 presents the behaviour of the material, which suffers a loss of stiffness. The darkened areas shown in Fig. 1 represent thermodynamic forces of loss of stiffness $A^{d}$ and of gain of stiffness $A^{h}$.

The effective tension of the material is defined by $\tilde{\boldsymbol{\sigma}}_{x}=E_{0} \boldsymbol{\varepsilon}_{x}$

\subsubsection{Stage of material gain of stiffness}

In the stage of gain of stiffness, there is material reparation, therefore: $\alpha^{d}=\alpha_{\text {crit }}^{d}$ and $\alpha^{h} \neq 0$. The critical damage, $\alpha_{\text {crit }}^{d}$, was adopted as 0,67 . The diagram that shows the behaviour of the material, which was previously damaged and is gaining stiffness, is shown 


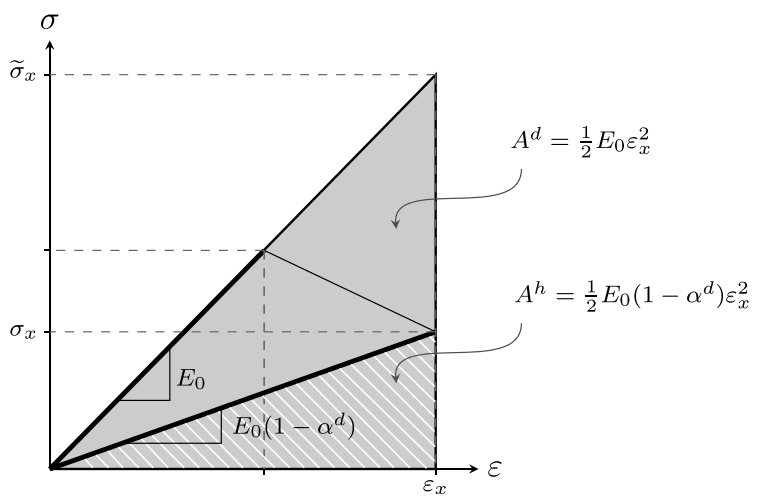

Fig. 1. Stress-strain diagram for the material at the stage of loss of stiffness.

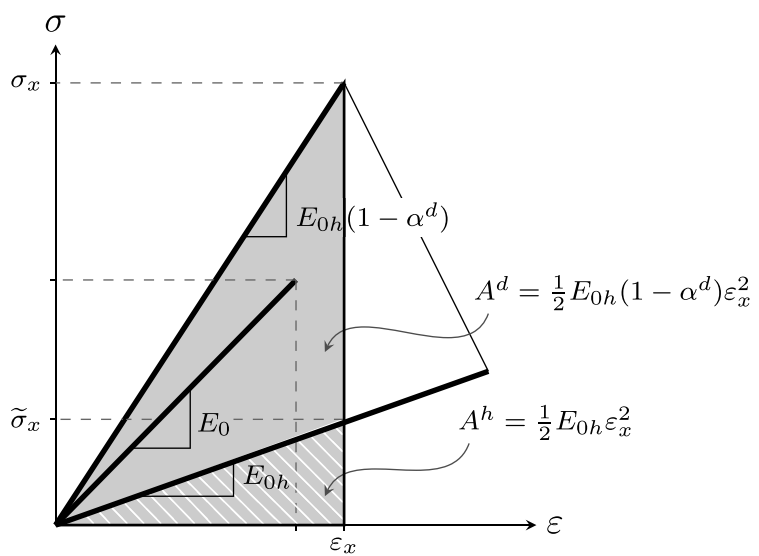

Fig. 2. Stress-strain diagram for the material at the stage of gain of stiffness.

in Fig. 2. The darkened area in Fig. 2 represents the thermodynamic forces of loss of stiffness $A^{d}$ and of gain of stiffness $A^{h}$.

The effective stress of material is defined as $\tilde{\boldsymbol{\sigma}}_{x}=$ $E_{0}^{h} \boldsymbol{\varepsilon}_{x}$, where $E_{0}^{h}=E_{0}\left(1-\alpha_{\text {crit }}^{d}\right)$ is Young's modulus of the bone in the moment it starts the gain of stiffness, in other words, the variable of loss of stiffness reaches the maximum value.

Figure 3 shows the admissible region $P_{A}$ as a projection of the admissible region $P$ in the thermodynamic forces space and also the sub-differential of the indicator function of region $P_{A}$. Region $P_{A}$ is the region of admissible thermodynamic forces.

For the one-dimensional problem, the formulation can be written considering the following:

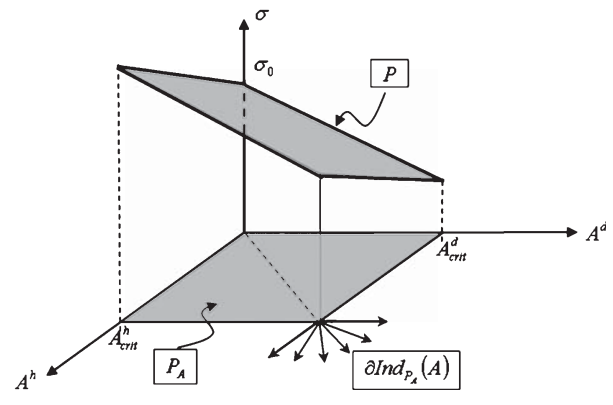

Fig. 3. Generalized stress diagram indicating the thermodynamically admissible regions for the problem.

$$
\begin{aligned}
\boldsymbol{D}\left(\alpha^{d}, a^{h}\right) & \equiv E_{0}\left(1-\alpha^{d}\right)^{n_{d}}\left(1+\alpha^{h}\right)^{n_{h}} \\
\boldsymbol{\varepsilon} & \equiv \boldsymbol{\varepsilon}_{x}
\end{aligned}
$$

where $E_{0}$ is Young's modulus for undamaged materials, $n_{d}$ is the exponent of damage, $n_{h}$ is the exponent of recuperation and $\boldsymbol{\varepsilon}_{x} \in \mathbb{R}$ is the longitudinal deformation.

In a similar study [11], the variables of loss and gain were defined with a unitary exponent. That consideration, associated with the results described in [42], justified the choice of $n_{d}=n_{h}=1$. As a result, it follows that:

$$
\begin{gathered}
\boldsymbol{D}\left(\alpha^{d}, \alpha^{h}\right)=E_{0}\left(1-\alpha^{d}\right)\left(1+\alpha^{h}\right) \\
\psi\left(\boldsymbol{\varepsilon}_{x}, \alpha^{d}, \alpha^{h}\right)=\frac{1}{2 \rho} E_{0}\left(1-\alpha^{d}\right)\left(1+\alpha^{h}\right) \boldsymbol{\varepsilon}_{x}^{2} \\
\boldsymbol{\sigma}_{x}\left(\boldsymbol{\varepsilon}_{x}, \alpha^{d}, \alpha^{h}\right)=E_{0}\left(1-\alpha^{d}\right)\left(1+\alpha^{h}\right) \boldsymbol{\varepsilon}_{x} \\
A^{d}\left(\boldsymbol{\varepsilon}_{x}, \alpha^{d}, \alpha^{h}\right)=\frac{1}{2} E_{0}\left(1+\alpha^{h}\right) \boldsymbol{\varepsilon}_{x}^{2} \\
A^{h}\left(\boldsymbol{\varepsilon}_{x}, \alpha^{d}, \alpha^{h}\right)=\frac{1}{2} E_{0}\left(1-\alpha^{d}\right) \boldsymbol{\varepsilon}_{x}^{2}
\end{gathered}
$$

In order to define the dissipation potential, it is necessary to determine the complementary potential (Gibbs free energy density) as a function of dual variables. Thus, substituting Equation 34 to Equation 7, one can obtain the complementary potential:

$$
\begin{aligned}
\frac{1}{\rho} \psi_{C}\left(\boldsymbol{\sigma}, A^{d}, A^{h}\right)= & \sup _{\boldsymbol{\varepsilon}, \alpha^{d} \alpha^{h}}\left[\boldsymbol{\sigma}_{x} \boldsymbol{\varepsilon}_{x}-A^{d} \alpha^{d}+A^{h} \alpha^{h}\right. \\
& \left.-\frac{1}{2 \rho} E_{0}\left(1-\alpha^{d}\right)\left(1+\alpha^{h}\right) \boldsymbol{\varepsilon}_{x}^{2}\right]
\end{aligned}
$$


Alternatively, as a function of dual variables, for a time $t$, it follows that:

$$
\begin{aligned}
& \frac{1}{\rho} \psi_{C}\left(\boldsymbol{\sigma}, A^{d}, A^{h}\right) \\
& \quad=\sup _{\boldsymbol{\varepsilon}, \alpha^{d}, \alpha^{h}}\left[\frac{3}{\sqrt[3]{2 E_{0}}}\left(\sigma_{x}^{2} A^{d} A^{h}\right)^{\frac{1}{3}}-A^{d}-A^{h}\right]
\end{aligned}
$$

Additionally, for a time $t+\Delta t$, that becomes:

$$
\begin{aligned}
& \frac{1}{\rho} \psi_{C}\left(\boldsymbol{\sigma}+\Delta \boldsymbol{\sigma}, A^{d}+\Delta A^{d}, A^{h}+\Delta A^{h}\right) \\
& =\sup _{\boldsymbol{\varepsilon}+\Delta \boldsymbol{\varepsilon}, \alpha^{d}+\Delta \alpha^{d}, \alpha^{h}+\Delta \alpha^{h}} \\
& \quad\left\{\frac{3}{\sqrt[3]{2 E_{0}}}\left[\left(\sigma_{x}+\Delta \sigma_{x}\right)^{2}\left(A^{d}+\Delta A^{d}\right)\left(A^{h}+\Delta A^{h}\right)\right]^{\frac{1}{3}}\right. \\
& \left.\quad-\left(A^{d}+\Delta A^{d}\right)-\left(A^{h}+\Delta A^{h}\right)\right\}
\end{aligned}
$$

According to Equation 25 and considering that from now on potential $\psi_{C}$ is the free energy per density, the dissipation potential in incremental form is defined by the following:

$$
\begin{aligned}
j_{C}( & \left.\Delta \boldsymbol{\sigma}, \Delta A^{d}, \Delta A^{h}\right) \\
= & \psi_{C}\left(\sigma+\Delta \boldsymbol{\sigma}, A^{d}+\Delta A^{d}, A^{h}+\Delta A^{h}\right) \\
& -\Delta \boldsymbol{\sigma} \cdot \nabla_{\boldsymbol{\sigma}} \psi_{C}\left(\boldsymbol{\sigma}, A^{d}, A^{h}\right) \\
& -\Delta A^{d} \cdot \nabla_{A^{d}} \psi_{C}\left(\boldsymbol{\sigma}, A^{d}, A^{h}\right) \\
& -\Delta A^{h} \cdot \nabla_{A^{h}} \psi_{C}\left(\sigma, A^{d}, A^{h}\right) \\
& -\psi_{C}\left(\boldsymbol{\sigma}, A^{d}, A^{h}\right)
\end{aligned}
$$

Applying the Convex Analysis definitions [26], potential $j_{C}$, defined as a function of generalised thermodynamic forces (dual variables), is established by the following:

$$
\begin{aligned}
j_{C}( & \left.\Delta \boldsymbol{\sigma}, \Delta A^{d}, \Delta A^{h}\right) \\
= & \frac{3}{\sqrt[3]{2 E_{0}}}\left(\sigma_{x}+\Delta \sigma_{x}\right)^{\frac{2}{3}}\left(A^{d}+\Delta A^{d}\right)^{\frac{1}{3}}\left(A^{h}+\Delta A^{h}\right)^{\frac{1}{3}} \\
& -\left(A^{d}+\Delta A^{d}\right)-\left(A^{h}+\Delta A^{h}\right) \\
& -\Delta \sigma_{x} \frac{2}{\sqrt[3]{2 E_{0}}} \sigma_{x}^{-\frac{1}{3}}\left(A^{d}\right)^{\frac{1}{3}}\left(A^{h}\right)^{\frac{1}{3}} \\
& -\Delta A^{d}\left[\frac{1}{\sqrt[3]{2 E_{0}}} \sigma_{x}^{\frac{2}{3}}\left(A^{d}\right)^{-\frac{2}{3}}\left(A^{h}\right)^{\frac{1}{3}}-1\right]
\end{aligned}
$$

$$
\begin{aligned}
& -\Delta A^{h}\left[\frac{1}{\sqrt[3]{2 E_{0}}} \boldsymbol{\sigma}_{x}^{\frac{2}{3}}\left(A^{d}\right)^{\frac{1}{3}}\left(A^{h}\right)^{-\frac{2}{3}}-1\right] \\
& -\frac{3}{\sqrt[3]{2 E_{0}}} \boldsymbol{\sigma}_{x}^{\frac{2}{3}}\left(A^{d}\right)^{\frac{1}{3}}\left(A^{h}\right)^{\frac{1}{3}}-A^{d}-A^{h}
\end{aligned}
$$

The increments of internal variables are written as follows:

$$
\begin{aligned}
& \Delta \alpha^{d}=\nabla_{\Delta A^{d} j_{C}}\left(\Delta \sigma, \Delta A^{d}, \Delta A^{h}\right) \\
& \Delta \alpha^{h}=\nabla_{\Delta A^{h} j_{C}}\left(\Delta \sigma, \Delta A^{d}, \Delta A^{h}\right)
\end{aligned}
$$

For the one-dimensional case, it becomes the following:

$$
\begin{aligned}
& \Delta \alpha^{d} \\
& =\frac{3}{\sqrt[3]{2 E_{0}}}\left[\left(\sigma_{x}+\Delta \sigma_{x}\right)^{\frac{2}{3}}\left(A^{d}+\Delta A^{d}\right)^{-\frac{2}{3}}\right. \\
& \\
& \left.\quad\left(A^{h}+\Delta A^{h}\right)^{\frac{1}{3}}-\sigma_{x}^{\frac{2}{3}}\left(A^{d}\right)^{-\frac{2}{3}}\left(A^{h}\right)^{\frac{1}{3}}\right](45) \\
& \begin{aligned}
\Delta \alpha^{h} \\
=\frac{3}{\sqrt[3]{2 E_{0}}}\left[\left(\sigma_{x}+\Delta \sigma_{x}\right)^{\frac{2}{3}}\left(A^{d}+\Delta A^{d}\right)^{\frac{1}{3}}\right. \\
\\
\left.\quad\left(A^{h}+\Delta A^{h}\right)^{-\frac{2}{3}}-\sigma_{x}^{\frac{2}{3}}\left(A^{d}\right)^{\frac{1}{3}}\left(A^{h}\right)^{-\frac{2}{3}}\right](46)
\end{aligned}
\end{aligned}
$$

The nonlinear programming problem can be formulated according to function $f$. If the remodelling function is negative and different from zero, $f\left(\sigma, A^{d}, A^{h}\right)<0$, then $\alpha^{d}=\alpha^{d}=0$ and no optimisation needs to be performed. If $f\left(\sigma, A^{d}, A^{h}\right)=0$ then the internal variables (restrictions) must be different from zero, $\alpha^{d}>0$ and $\alpha^{h}>0$, the values of $\alpha^{d}$ and $\alpha^{h}$ are found by optimisation. The objective function $f$ is never greater than zero.

\subsection{One-dimensional example of a bar that represents the bone sample}

The proposed example represents one bar (an experimental sample of a bone trabecula), shown in Fig. 4. This example is comprised by two one-dimensional elements, as shown in Fig. 5, which are under the influence of a growing load cycle. This dynamic process can represent the rehabilitation of an athlete, starting with light exercises up to a heavy physical activity. In this context, one load cycle represents a day of exercises. 


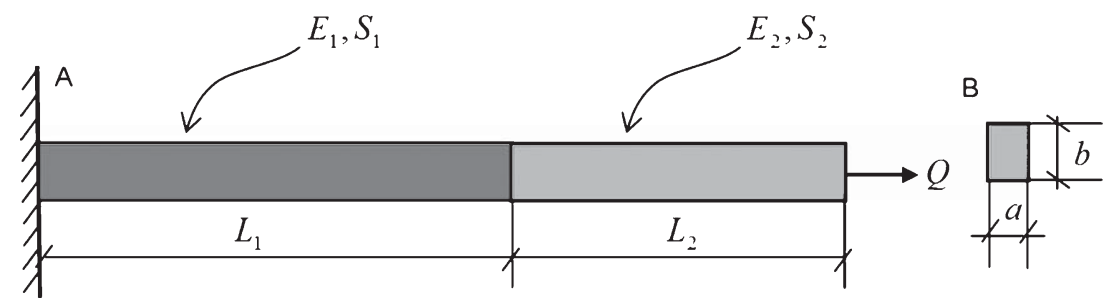

Fig. 4. (A) Bone trabecula formed by two elements, (B) width and thickness represented by letters $a$ and $b$.

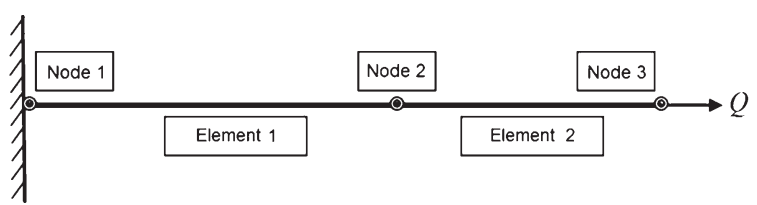

Fig. 5. Definition of the elements and nodes for the one-dimensional geometry of a trabecula model.

For the numerical solution of the problem, the REMOLD 1D program was developed and implemented using the Galerkin spatial discretization and rigidity matrices. This program solves the global equilibrium of forces through the Quasi-Newton Method and the constitutive problem (the evolution of internal variables) through the Newton-Raphson Method.

The compiler used was GFortran 4.1 (GNU Fortran compiler) and the programming language of the code is Fortran 90. The Gnuplot 4.2 program was used for the visualisation of the results.

The remodelling function used for the onedimensional example has the form:

$$
f\left(\sigma, A^{d}, A^{h}\right)=\sigma_{x}+\left(A^{d}-A_{0}^{d}\right)+\left(A^{h}-A_{0}^{h}\right) \leq 0
$$

Equation 47 is one adaptation of the Drucker-Prager model for the uniaxial stress state, as established in study [30].

Two simulations were performed: first, both elements of the bar were considered initially with the same rigidity, and then, there was one element with initial damage. The results of the simulations described above were compared with the experimental results of [8].

Table 1 shows the data for both simulation schemes (without initial damage and with initial damage in one element).The numbers of the elements and the nodes of the model are drawn in Fig. 5. Bones are anisotropic and heterogeneous structures, and the differences in
Table 1

Data of the Model

\begin{tabular}{lll}
\hline Data & Element 1 & Element 2 \\
\hline Length $L(\mathrm{~m})$ & 0,015 & 0,015 \\
Height $b(\mathrm{~m})$ & 0,01 & 0,01 \\
Thickness $a(\mathrm{~m})$ & 0,01 & 0,01 \\
Young's modulus $E(\mathrm{MPa})$ & 450 & 450 \\
Maximum of $\alpha^{d}=\alpha_{\text {crit }}^{d}$ & 0,67 & 0,67 \\
Maximum of $\alpha^{h}=\alpha_{\text {crit }}^{h}$ & 1,0 & 1,0 \\
Thermodynamic force $A_{0}^{d}(\mathrm{~Pa})$ & 969,61 & 969,61 \\
Thermodynamic force $A_{0}^{h}(\mathrm{~Pa})$ & 10000,0 & 10000,0 \\
\hline
\end{tabular}

the level of damage in each element play an important role in distinct stages of the remodelling process.

The total number of load cycles is 1200; each load cycle has only one load step and the final load applied in the bar is $350 \mathrm{~N}$. The loads start from zero and increase in equal increments for each load cycle up to day 1200 .

\section{Results}

\subsection{Initially undamaged material}

Figure 6 illustrates the stress-strain diagrams for both elements that are considered to be initially undamaged. Those results were obtained for 1200 load cycles in the REMOLD 1D program. The load of each load cycle grows over time; therefore the load of a subsequent load cycle is always greater than the previous cycle. There is only one load step in each load cycle.

The evolution of the internal scalar variables of bone remodelling, $\alpha^{d}$ and $\alpha^{h}$, can be found in Fig. 7. The results are shown up to the 1000 load cycle. Figure 8 shows the evolution of Young's modulus.

\subsection{Initially damaged material for element 2}

In this case, an initial value of $10 \%$ for $\alpha^{d}$ (the loss of stiffness variable) of element 2 was 

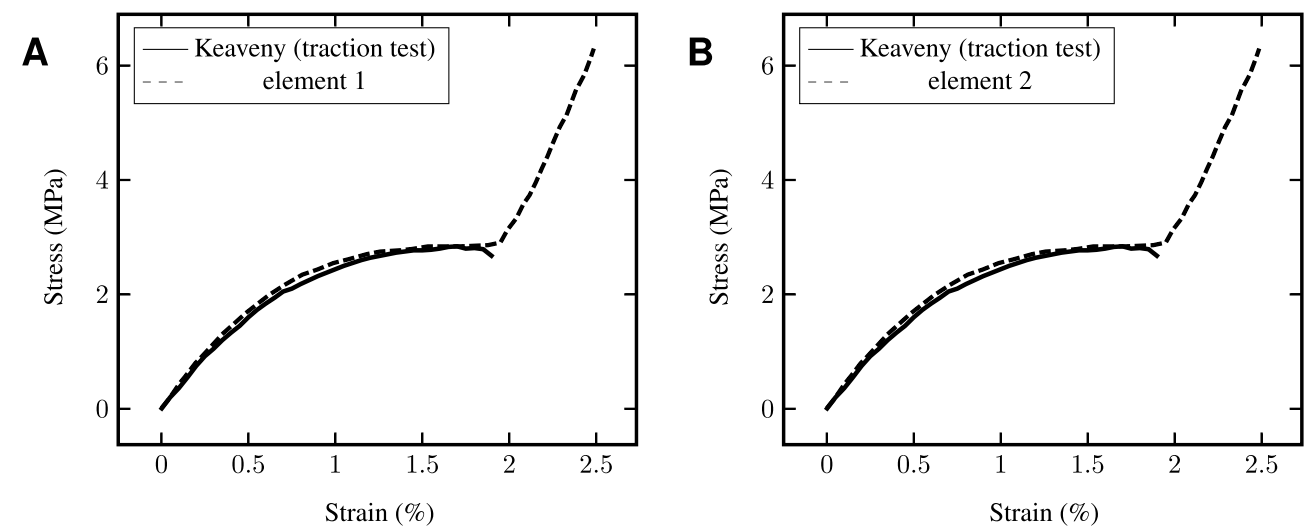

Fig. 6. Stress-strain diagrams for element 1 (A) and element 2 (B).
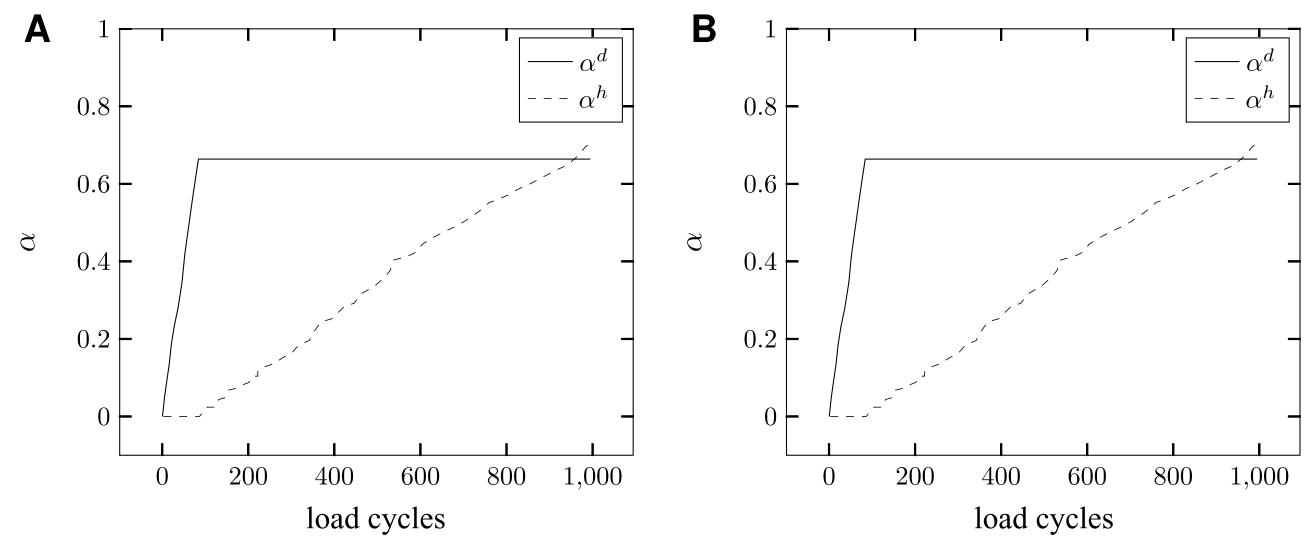

Fig. 7. Evolution of internal variables $\left(\alpha^{d}\right)$ and $\left(\alpha^{h}\right)$ for element 1 (A) and element 2 (B) along the load cycles.
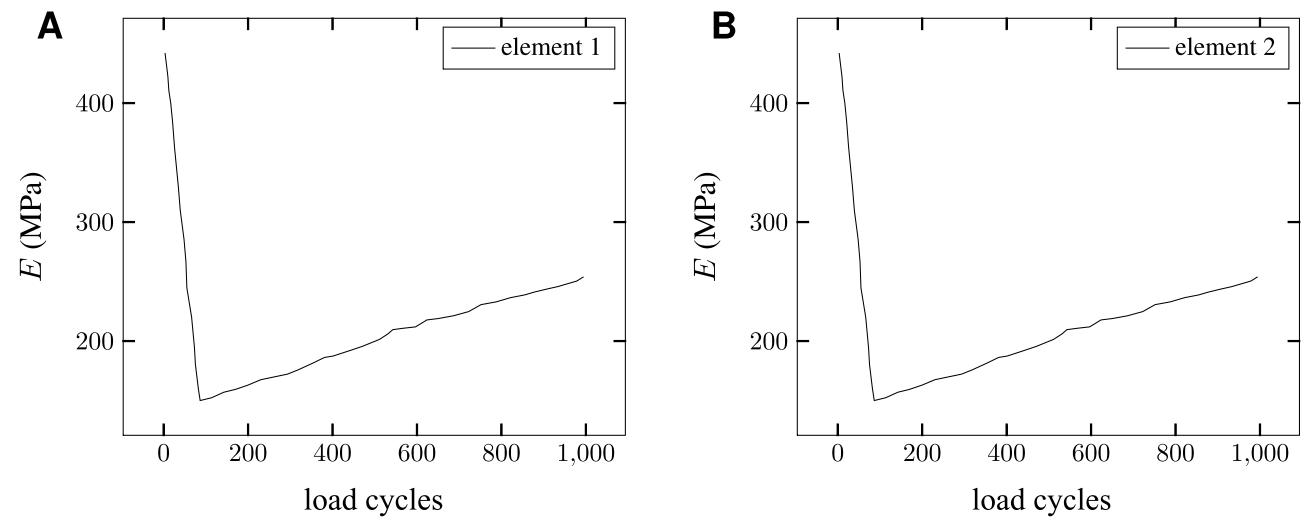

Fig. 8. Evolution of Young's modulus for element 1 (A) and element 2 (B) along the load cycles.

established. The internal variable $\alpha^{d}$ for element 1 is zero at the beginning of the simulation. For this example, the program processed 1200 load cycles.
The results obtained for the stress-strain diagram are illustrated in Fig. 9. Figures 10 and 11 show the evolution of internal variables and Young's modulus, respectively. 


\section{Discussion}

\subsection{Initially undamaged material}

This example shows how the remodelling process in the trabecula is processed. After a damage or loss of stiffness process, the biological system acts in a way to restore the initial stiffness.

Figure 6 shows that the obtained result was very close to the result described by [8] in terms of the initial elasticity (linear) and loss of stiffness (non-linear). For deformation levels greater than $2.0 \%$, the stage of gain of stiffness begins. In this stage there are no experimental results for comparison yet. Due to this scenario, the authors adopted some parameters that needed to be calibrated according to experimental results regarding bone formation.

In Fig. 7, when the loss of stiffness variable $\alpha^{d}$ reaches its maximum value, 0.67 , the damage is maintained and the gain process begins from the damaged material.

Near load cycle 1000 (day 1000), the value of the gain of stiffness variable $\alpha^{h}$ overcomes the value of the loss of stiffness variable, but this scenario does not indicate that the material reaches its initial Young's modulus value, as it is shown in Fig. 8.

Figure 8 shows that the loss of stiffness occurs until approximately day 100, when the gain of stiffness process begins, at a lower rate.

The stage of loss of stiffness may represent the activity of the osteoclasts (removing bone matrix) or the mechanical damage. The stage of gain of stiffness represents the activity of the osteoblasts (forming new bone). The example shows the evolution of the elastic- ity modulus in one cycle of bone remodelling for the trabecula.

\subsection{Initially damaged material for element 2}

The purpose of this example is to analyse how different parts of the same trabecula model behave with different initial conditions. While in the previous example both elements had the same remodelling results, in this example element 2 starts the stage of gain of stiffness before element 1 .

The stress $\times$ strain diagram of both elements (Fig. 9) is similar to the results of [8]; element 2 presents lower stress levels compared to element 1 in the stage of loss of stiffness. The stage of gain of stiffness begins from a lower deformation level (between $1.5 \%$ and $2.0 \%$ ) in element 2 and finishes with a higher level of stress. This scenario was expected because the damage was set to begin in element 2 first.

By the end of the remodelling process, at load cycle 750 (Fig. 10), the value of $\alpha^{h}$ for element 2 is higher than for element 1 . This can be explained by the initial conditions, element 2 reaches maximum damage level before element 1 , so it starts the stage of gain of stiffness before element 1 and grows before too.

The Young's modulus of element 2 begins with a smaller value compared to element 1 . Afterwards, it decreases in the stage of loss of stiffness (up to day 100) and increases in the stage of gain of stiffness, as it is expected and shown in Fig. 11. The remodelling process occurs before in the initially damaged element.
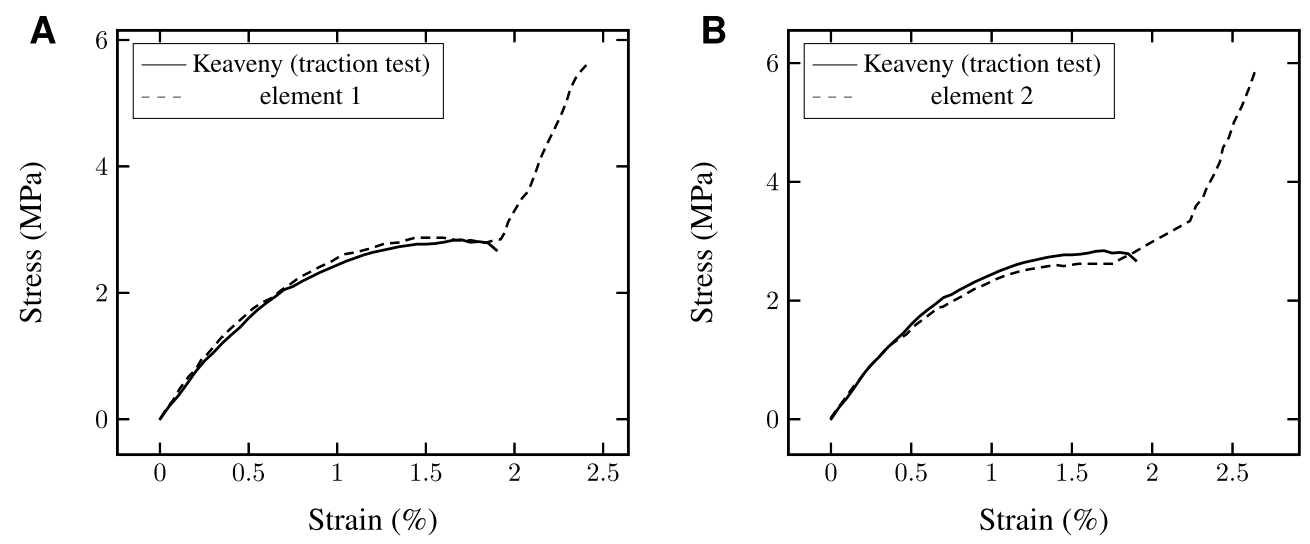

Fig. 9. Results of stress-strain diagrams for element 1 (A) and element 2 (B) - initial damage. 

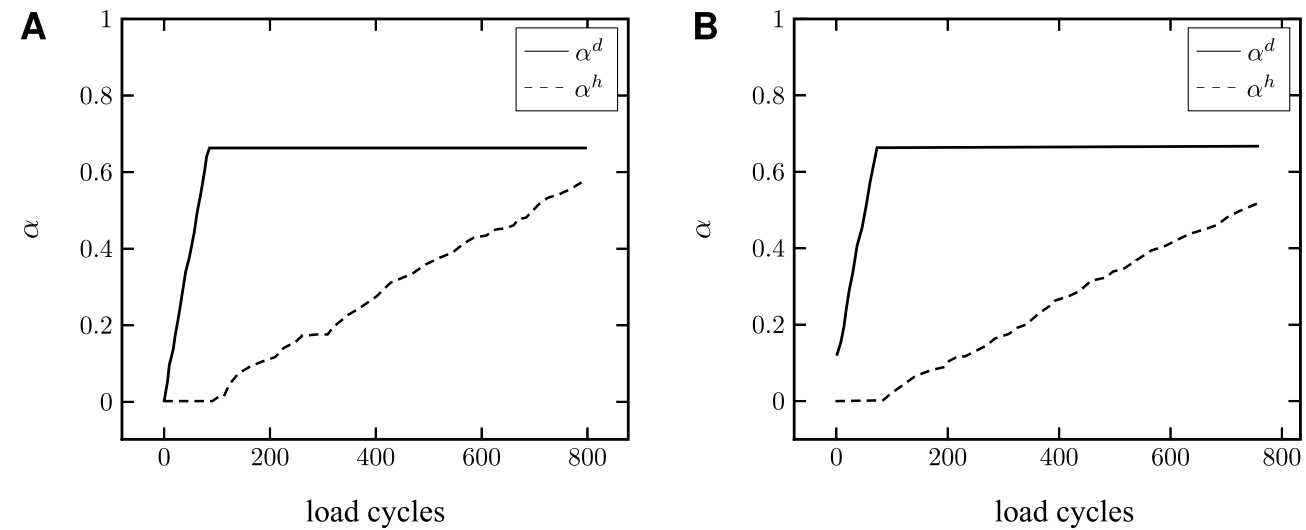

Fig. 10. Evolution of internal variables $\left(\alpha^{d}\right)$ and $\left(\alpha^{h}\right)$ for element 1 (A) and for element 2 (B) along the load cycles - initial damage.
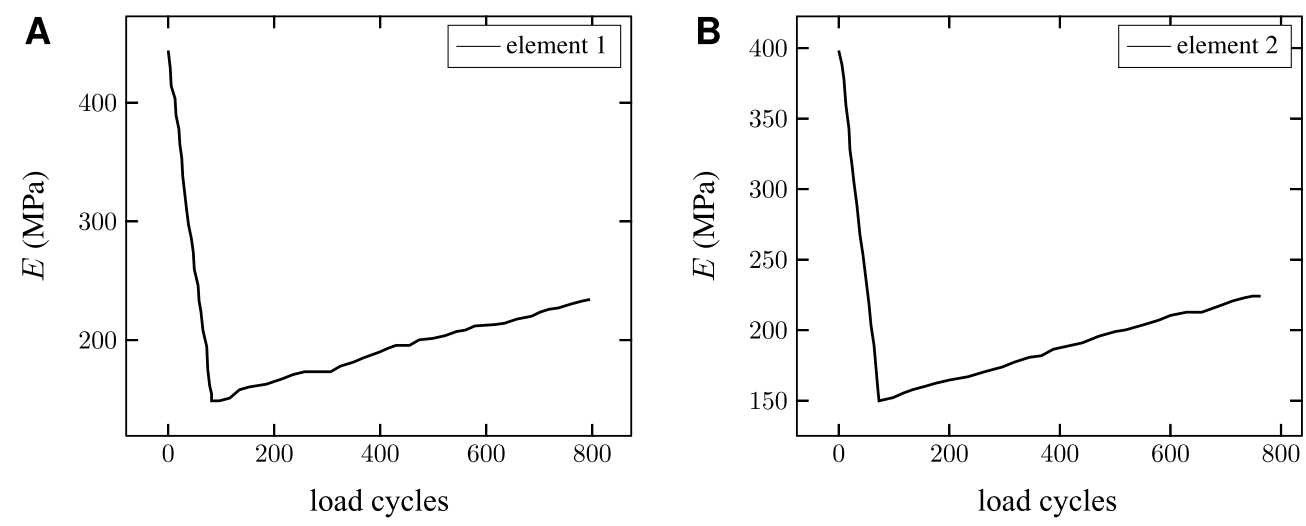

Fig. 11. Evolution of Young's modulus for element 1 (A) and element 2 (B) along the load cycles - initial damage.

\section{Conclusion}

This study presents a consistent thermodynamic formalism for bone remodelling, which may allow further contributions, such as the incorporation of chemical reactions, mass transference, anisotropic damage and other significant characteristics of this complex natural phenomenon. The constitutive equations derived from state equations are more reliable compared to the empiric approaches, because the former automatically satisfy the thermodynamic restrictions.

This framework also allows the reproduction of other constitutive model formulations that are found in the literature, depending on the appropriate choice of internal variables and on the kind of constitutive potential.

Nowadays, there is little equipment that can have access to in vivo data that could be used to estimate bone density history during remodelling. However, a mechanical test can give some insights as to whether the model is able to respond to a varying time load case or not, especially in the damage zone. The presented model was calibrated for a traction experiment of a bone specimen.

The proposed theory was applied to a onedimensional finite element example, and two situations (an undamaged material and an initially damaged material) were compared to the experiment curve. Focusing the comparison only on the part of the loss of stiffness (before load cycle 100) the presented stress $x$ strain curves showed accordance to the experiment results.

Figures 7 and 8, respectively, show the evolution of the internal variables and Young's Modulus concerning load cycles in the initially undamaged simulation, respectively. It can be seen that when $\alpha^{h}=\alpha_{\text {crit }}^{d}$ the elasticity modulus does not reach the original value. This can be explained by the choice of the Helmholtz 
free energy potential and subsequent derivation of the thermodynamic forces. A different formulation that will respond equally during the stages of loss and gain of stiffness is shown below:

$$
\psi\left(\boldsymbol{\varepsilon}_{x}, \alpha^{d}, \alpha^{h}\right)=\frac{1}{2 \rho} E_{0}\left(1-\alpha^{d}+\alpha^{h}\right) \varepsilon_{x}^{2}
$$

The aim of the simulation was achieved with the application of the REMOLD 1D program. Nevertheless, further developments can be studied such as other propositions for the Helmholtz free energy potentials and the extension to two and three-dimensional cases. Next steps for the research should also include different failure criteria for bone [61] and the differences between the tensile and compressive strengths of bone [60].

\section{Acknowledgments}

This study was funded in part by the Coordination for the Improvement of the Higher Level Education Personnel (CAPES) from the Brazilian Federal Government. Scholarships for master and doctoral courses were granted to the first two authors.

\section{References}

[1] A. Benallal, Continuous Damage and Fracture, Elsevier, 2000.

[2] A.G. Robling, A.B. Castillo and C.H. Turner, Biomechanical and molecular regulation of bone remodeling, Annual Review of Biomedical Engineering 8 (2006), 455-498.

[3] B.D. Reddy and J.B. Martin, Algorithms for the solution of internal variable problems in plasticity, Comp Meths Appl Mech Eng 93 (1991), 253-273.

[4] C. Verna, M. Dalstra, T.C. Lee, P.M. Cattaneo and B. Melsen, Microcracks in the alveolar bone following orthodontic tooth movement: A morphological and morphometric study, Composites Science and Technology 26 (2004), 459-467.

[5] C.H. Turner, Three rules for bone adaptation to mechanical stimuli, Bone 23(5) (1998), 399-407.

[6] C.R. Jacobs, J.C. Simo, G.S. Beaupre and D.R. Carter, Adaptive bone remodeling incorporating simultaneous density and anisotropy considerations, Journal of Biomechanics 30(6) (1997), 603-613.

[7] D.B. Burr, Damage detection and behavior in bone, In: Proceedings of the 12th Conference of the European Society of Biomechanics, Dublin, 2000, pp. 38-39.

[8] D.L. Kopperdahl and T.M. Keaveny, Yield strain behavior of trabecular bone, Journal of Biomechanics 31 (1998), 601-608.

[9] E. Fancello, J.P. Ponthot and L. Stainier, A variational formulation of constitutive models and updates in non-linear finite viscoelasticity, International Journal for Numerical Methods in Engineering 65 (2006), 1831-1864.

[10] E.G.F. Mercuri, M.B. Hecke, L.Z. Largura and F.V. Tormena, Modelo computacional para descrever o remodelamento ósseo aplicado ao estudo ortodôntico usando potenciais termodinâmicos generalizados (Quoted in Portuguese) [Computational model for bone remodeling using thermodinamic potentials applied to orthodontic movement]. Proceedings of Iberian Latin American Congress on Computational Methods in Engineering (XXIX CILAMCE). Federal University of Alagoas, Maceió, 2008.

[11] E.J. Barbero, F. Greco and P. Lonetti, Continuum damage-healing mechanics with application to self-healing composites, International Journal of Damage Mechanics 14 (2005), 51-81.

[12] E.K. Ncheuguim, Theoretical aspects of classical and Drucker-Prager cap models of elastoplasticity, AIMS Essay, 2006.

[13] G. Bodovillé and G. Saxcé, Plasticity with non-linear kinematic hardening: Modeling and shakedown analysis by the bipotential approach, Eur J Mech A/Solids 20 (2001), 99112.

[14] G. Maier, Mathematical programming methods in structural analysis, In: Proceedings of the International Symposium on Variational Methods in Engineering (vol. 8), Southampton University Press, 1973, pp. 1-13.

[15] G.D. Nguyen, A Thermodynamic Approach to Constitutive Modelling of Concrete using Damage Mechanics and Plasticity Theory, Trinity College, University of Oxford, London, 2005.

[16] G.T. Houlsby, Some Mathematics for the Constitutive Modelling of Soils, in Proceedings of the Geomath Symposium, Horton, Greece, 2002.

[17] H. Weinans, R. Huiskes and H. Grootenboer, The behavior of adaptive bone-remodeling simulation models, Journal of Biomechanics 25(12) (1992), 1425-1441.

[18] H.H. Bayraktar, E.F. Morgan, G.L. Niebur, G.E. Morris, E.K. Wong and T.M. Keaveny, Comparison of the elastic and yield properties of human femoral trabecular and cortical bone tissue, Journal of Biomechanics 37 (2004), 27-35.

[19] H.M. Frost, Skeletal structural adaptations to mechanical usage (SATMU): 1. Redefining Wolff's Law: The bone modeling problem, The Anatomical Record 226(4) (1990), 403-413.

[20] H.M. Frost, Skeletal structural adaptations to mechanical usage (SATMU): 2. Redefining Wolff's Law: The remodeling problem, The Anatomical Record 226(4) (1990), 414422.

[21] H.M. Frost, Vital biomechanics: Proposed general concepts for skeletal adaptations to mechanical usage, Calcified Tissue International 42 (1988), 145-156.

[22] I. Einav, G.T. Houlsby and G.D. Nguyen, Coupled damage and plasticity models derived from energy and dissipation potentials, International Journal of Solids and Structures $\mathbf{4 4}$ (2007), 2487-2508.

[23] J. García-Aznar, T. Rueberg and M. Doblare, A bone remodeling model coupling microdamage growth and repair by $3 \mathrm{D}$ BMU-activity, Biomechanics and Modeling in Mechanobiology 4 (2005), 147-167.

[24] J. Lemaitre and J.L. Chaboche, Mechanics of Solid Materials, Cambridge: Cambridge University Press, 1990.

[25] J. Lemaitre, A Course on Damage Mechanics, 2 ed., Heidelberg: Springer-Verlag, 1996. 
[26] J.B. Hirriart-Urruty and C. Lemarechal, Convex Analysis and Minimization Algorithms, Heidelberg: Springer-Verlag, 1993

[27] J.E. Shea and S.C. Miller, Skeletal function and structure: Implications for tissue-targeted therapeutics, Advanced Drug Delivery Reviews 57(7) (2005), 945-957.

[28] J.L. Chaboche, Cyclic viscoplastic constitutive equations, Part I: A thermodynamically consistent formulation, Part II: Stored energy comparison between models and experiments, Journal of Applied Mechanics 60 (1993), 822-828.

[29] K. Matsuo and N. Irie, Osteoclast-osteoblast communication, Archives of Biochemistry and Biophysics 473(2) (2008), 201-209.

[30] L. Contrafatto and M. Cuomo, A framework of elastic-plastic damaging model for concrete under multiaxial stress states, International Journal of Plasticity 22 (2006), 2272-2300.

[31] L. Contrafatto and M. Cuomo, A new thermodynamically consistent continuum model for hardening plasticity coupled with damage, Solids and Structures 39 (2002), 6241-6271.

[32] L. Rincón-Kohli and P.K. Zysset, Multi-axial mechanical properties of human trabecular bone, Biomechanics and Modeling in Mechanobiology 9 (2008), 195-208.

[33] L.M. McNamara and P.J. Prendergast, Bone remodeling algorithms incorporating both strain and microdamage stimuli, Journal of Biomechanics 40 (2007), 1381-1391.

[34] M. Buliga, G. Saxcé and C. Vallée, Existence and construction of bipotentials for graphs of multivalued laws, Journal of Convex Analysis 15 (2008), 87-104.

[35] M. Doblaré, J.M. García and M.J. Gómez, Modelling bone tissue fracture and healing: A review, Engineering Fracture Mechanics 71 (2004), 1809-1840.

[36] M. Hjiaj, M. Fortin and G.A. De Saxcé, Complete stress update algorithm for the non-associated Drucker-Prager model including treatment of the apex, International Journal of Engineering Science 41 (2003), 1109-1143.

[37] M.B. Hecke, L.M.R. Carvalho and J.A.O. Simões, A bone remodeling constitutive model using mathematical programming, In: Proceedings of the International Conference on Engineering Optimization, Rio de Janeiro, 2008.

[38] M.B. Hecke, Potenciais Generalizados e Otimização na Análise Elasto-Plástica (Quoted in Portuguese) [Generalized Potentials and Optimization in Elasto-Plastic analysis], Pontifícia Universidade Católica do Rio de Janeiro, Rio de Janeiro, 1991

[39] N. Zouain, M.B. Hecke and R.A. Feijóo, Elastic-Plastic Analysis with Potential Functionals using Internal Variables, in: Computational Plasticity: Fundamentals and Applications Proceedings of the Third International Conference, of: D.R.J. Owen, E. Oñate and E. Hilton, ed., Swansea: Pineridge Press, Barcelona, 1992, pp. 163-174.

[40] P. Zysset and L. Rincón-Kohli, An alternative fabric-based yield and failure criterion for trabecular bone, in: Mechanics of Biological Tissue, G.A. Holzapfel and R.W. Ogden, ed., Springer Berlin Heidelberg, 2006, pp. 457-470.

[41] P.J. Prendergast and D. Taylor, Prediction of bone adaptation using damage accumulation, Journal of Biomechanics 27 (1994), 1067-1076.

[42] Q.D. Yang, B. Cox, R. Nalla and R. Ritchie, Fracture length scales in human cortical bone: The necessity of nonlinear fracture models, Biomaterials 27 (2006), 2095-2113.
[43] Q.S. Nguyen, Stability and Nonlinear Solid Mechanics, John Wiley and Sons Ltd, 2000.

[44] R. Huiskes, H. Weinans, H. Grootenboer, M. Dalstra, B. Fudala and T. Slooff, Adaptive bone-remodeling theory applied to prosthetic-design analysis, Journal of Biomechanics 20(11-12) (1987), 1135-1150.

[45] R. Ruimerman, Modeling and remodeling in bone tissue, TechnischeUniversiteit Eindhoven, 2005.

[46] R.A. Feijóo and N. Zouain, Elastic-plastic potential functionals for rates and increments of stress and strain, Com putational and Applied Mathematics 10 (1991), 207-228.

[47] R.A. Feijóo and N. Zouain, Formulations in rates and increments for elastic-plastic analysis, International Journal for Numerical Methods in Engineering 26 (1988), 2031-2048.

[48] R.A. Feijóo and N. Zouain, Variational Formulations for rates and increments in plasticity, In: Proceedings of the International Congress on Computational Plasticity (vol. 1), Barcelona, 1987.

[49] R.B. Martin and D.B. Burr, A hypothetical mechanism for the stimulation of osteonal remodeling by fatigue damage, Journal of Biomechanics 15 (1982), 137-139.

[50] R.B. Martin, Is all cortical bone remodeling initiated by microdamage? Bone 30 (2002), 8-13.

[51] R.B. Martin, Toward a unifying theory of bone remodeling, Bone 26 (2000), 1-6.

[52] R.T. Rockafellar, Convex Analysis, Princeton University Press, USA, 1973.

[53] S. Murakami and K. Kamiya, Constitutive and damage equations of elastic-brittle material based on irreversible thermodynamics, International Journal of Mechanical Sciences 39 (2007), 473-486

[54] S. Ramtani and M. Zidi, A theoretical model of the effect of continuum damage on a bone adaptation model, Journal of Biomechanics 34 (2001), 471-479.

[55] S. Ramtani and M. Zidi, Damage bone remodeling theory: Thermodynamical approach, Mechanics Research Communications 264(6) (1999), 701-708.

[56] S.C. Cowin and D.H. Hegedus, Bone remodeling I: Theory of adaptive elasticity, Journal of Elasticity 6 (1976), 313-326.

[57] S.C. Cowin, Wolff's law of trabecular architecture at remodeling equilibrium, Journal of Biomechanical Engineering 108(1) (1986), 83-88.

[58] S.J. Hazelwood, R.B. Martin, M.M. Rashid and J.J. Rodrigo, A mechanistic model for internal bone remodeling exhibits different dynamic responses in disuse and overload, Journal of Biomechanics 34 (2001), 299-308.

[59] T.C. Lee, A. Staines and D. Taylor, Bone adaptation to load Microdamage as a stimulus for bone remodeling, Journal of Anatomy 201 (2002), 437-446.

[60] T.M. Keaveny, E.F. Wachtel, C.M. Ford and W.C. Hayes, Differences between the tensile and compressive strengths of bovine tibial trabecular bone depend on modulus, Journal of Biomechanics 27 (1994), 1137-1146.

[61] T.M. Keaveny, E.F. Wachtel, S.P. Zadesky and Y.P. Arramon, Application of the Tsai-Wu quadratic multi-axial failure criterion to bovine trabecular bone, Journal of Biomechanics 121 (1999), 99-107.

[62] W. Han and B.D. Reddy, Plasticity - Mathematical Theory and Numerical Analysis, 2 ed., Springer-Verlag, New York, 1999. 

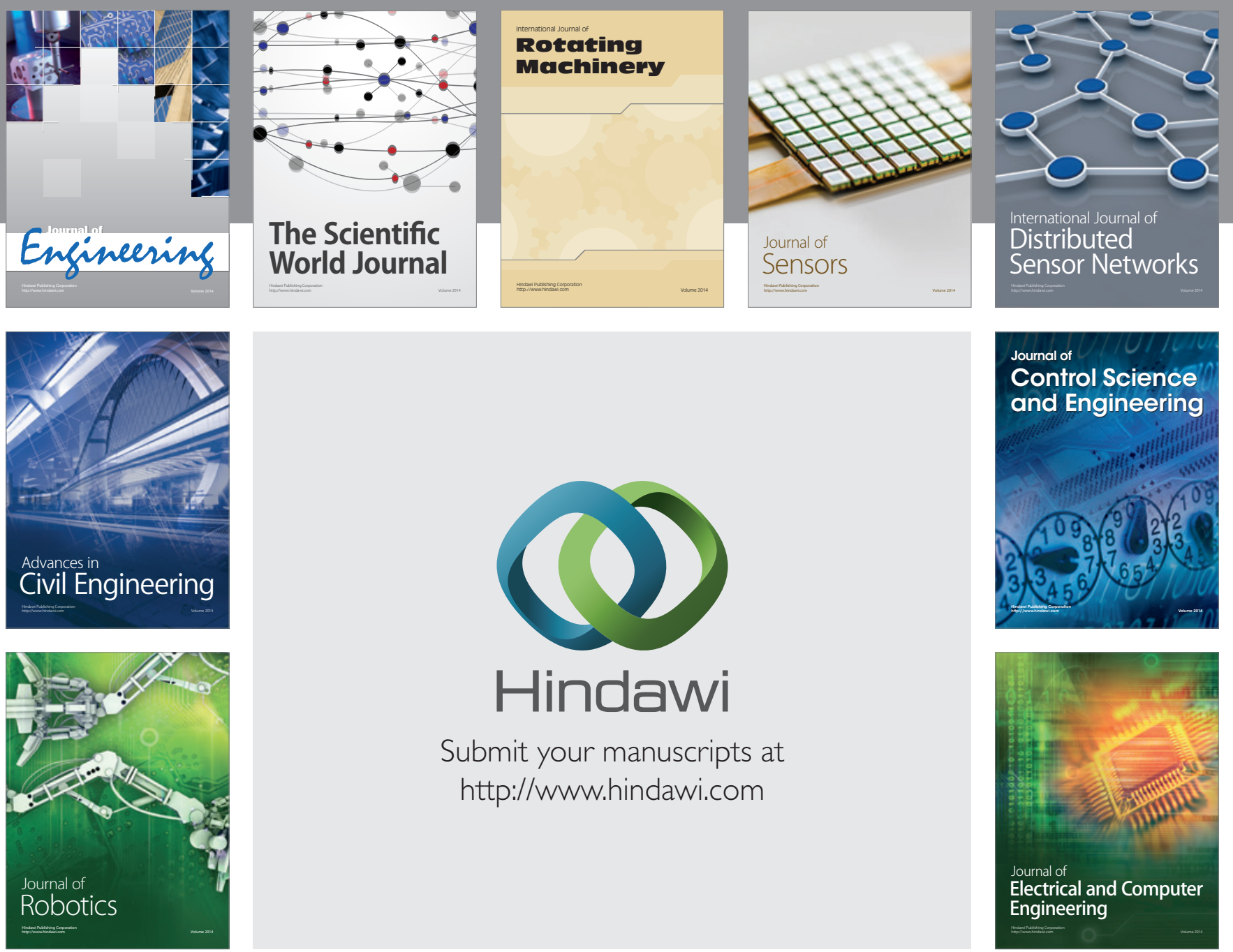

Submit your manuscripts at

http://www.hindawi.com
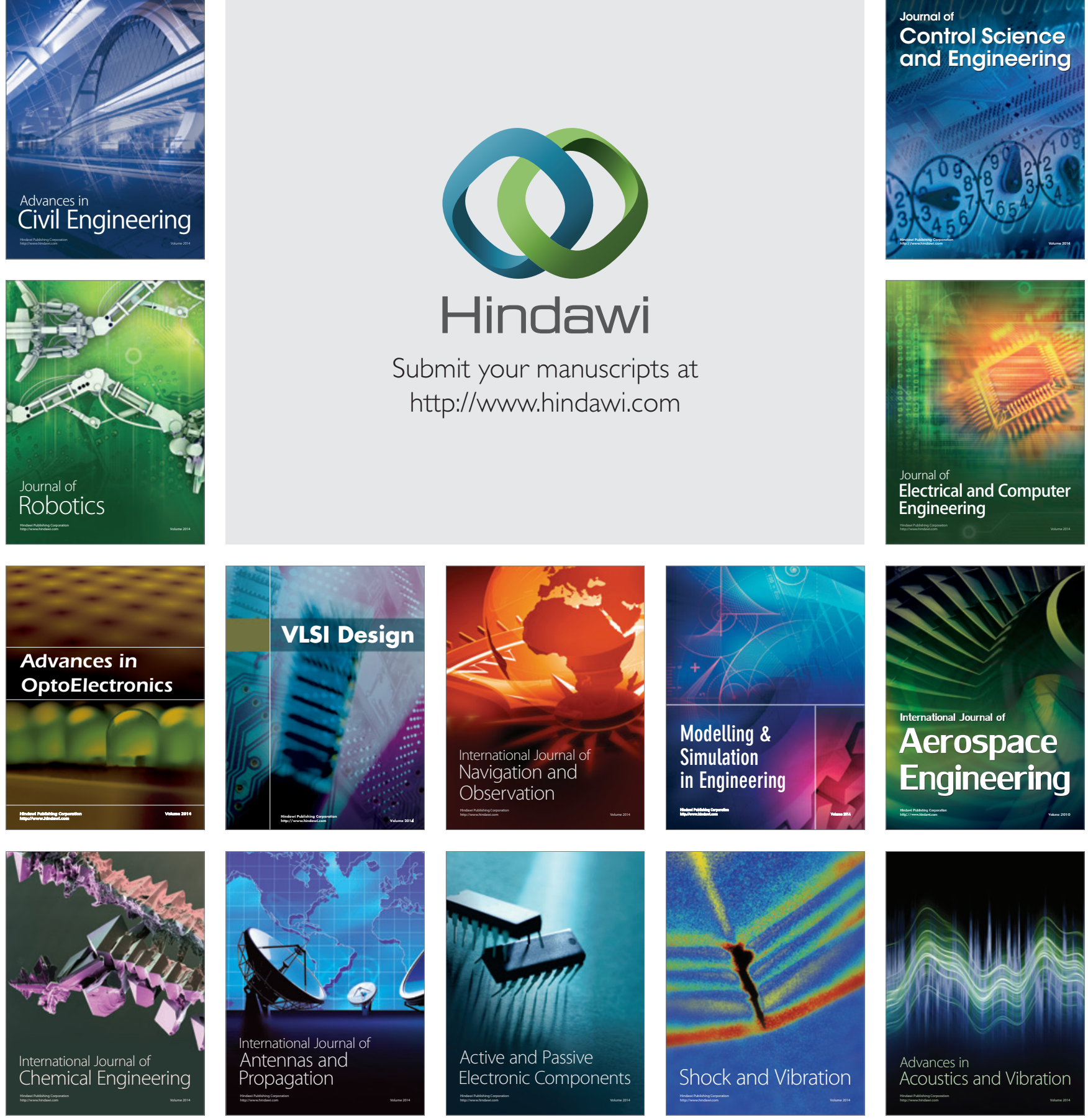